\title{
Sprouting Value Index and Viability Percentage: An Integrated Measure of Vegetative Planting Material
}

\author{
Justin R. Nayagam \\ Department of Botany, Union Christian College, Affiliated to Mahatma Gandhi University, Kerala 683102, India
}

\begin{abstract}
Rooting ability of indole 3-butyric acid (IBA)-induced stem cuttings in vegetative regeneration experiments depends on various parameters, which can be entrenched using sprouting value index (SVI), a mathematical approach. In this study, field studies were carried out in Tabebuia rosea to generate clonal planting stock regeneration using three rooting medium at a location in Central Kerala, Peninsular India, for three consecutive years from October 2014 till June 2016 at four months regular intervals. Three IBA concentrations, viz., 200, 500 and 1,000 ppm, have been used and the data obtained was evaluated using SVI method in order to establish the suitable medium, which gives the maximum rooting results. Control cuttings did not record rooting in any of the trials. The high SVI was obtained when stem root cuttings planted in root trainers with coir pith compost (RTCP) for all the three concentration of IBA applied. Sprouting percentage was marked up and the delay in completion of sprouting/rooting initiation decreased by the use of IBA treatment.
\end{abstract}

Key words: Pink trumpet tree, sprouting value index, rooting ability, indole 3-butyric acid.

\section{Introduction}

Artificial regeneration of economically important plants has a global effect, as it is a fundamental occupation of human kind and its discovery dates back to the origin of civilization and ornamental gardening $[1,2]$. The clonal regeneration of angiosperm plants occurs comparatively easily, as many of them produce bulbs, rhizomes, corms, tubers, offsets, suckers and stolon. While, vegetative regeneration in tree species is a difficult process and it is attained through rooting induction of stem cuttings using phytohormone application, such as indole 3 -acetic acid (IBA) and $\alpha$-naphthalene acetic acid [3-5]. There are several mathematical expressions to explain seed germination and seed characteristics in sexually reproducing plants [6-10]. Several experiments using different phyto-hormones are available in various plants with multifarious usage [11-15], but mathematical expressions for determining efficiency of vegetative planting material or for clonal

Corresponding author: Justin R. Nayagam, Ph.D., research field: plant physiology. propagation are scanty. The success of experimental design and its reliability lies in the success of statistical correlation and it leads to effective interpretation. Hence, the data obtained through the present investigation are being interpreted using sprouting value index proposed by Nayagam [16], as it gives idea on the efficiency of the media for planting, rooting success and the appropriate concentration of phytohormone which is beneficial.

\section{Materials and Methods}

\subsection{Specimen Collection}

For the present investigation, stem cuttings were procured from 10 year old Tabebuia rosea, the pink trumpet tree in the T.C Joseph Memorial Botanical Garden, Department of Botany, Union Christian College, Aluva, (107'30.65" N, 76²0'3.32" E) Ernakulam district, Kerala State, India. All field trials were conducted in the plant nursery of T.C Joseph Memorial Botanical Garden.

For the sake of large scale planting material production in mechanized gardens, trials were 
conducted in $100 \mathrm{cc}$ root-trainer blocks (24 celled) using potting mixture (RTPM), polyurethane foam cubes (PFC) and root trainers with coir pith (RTCP) three different rooting medium.

\subsection{Experiment Design}

The experiment was performed during October 2014 to February 2016 at four months regular intervals in three different rooting medium, such as RTPM, PFC and RTCP. Stem cuttings with an average size of $7.5 \mathrm{~cm}$ to $10 \mathrm{~cm}$ were used. Twenty-four stem cuttings were kept in each rooting medium separately, as the $100 \mathrm{cc}$ root-trainer block contains 24 cells. A non-auxin control and three IBA concentrations with 200, 500 and 1,000 ppm (parts per million) were designed in this experiment in order to detect the rooting/sprouting ability by quick dip method. A randomized complete block design was employed. After $45 \mathrm{~d}$, the cuttings were evaluated for rooting/sprouting percentages, mortality percentage and viability percentage. The data obtained were subjected to one factor analysis, employing analysis of variance (ANOVA) and two-way ANOVA.

\subsection{Calculation of Sprouting Value Index (SVI)}

In order to get SVI, data regarding sprouting and successful rooting percentages (SP), percentage of callus production without rooting (CWR), percentage of sound non-sprouted propagule without callus production (SUP) were determined by vertical cut test, i.e., planting material with more than three nodes living tissues was considered viable and prepared. Viability percentage (VP) can be determined using the following Eq. (1):

$$
\mathrm{VP}=\mathrm{SP}+\mathrm{CWR}+\mathrm{SUP}
$$

Peak value $(\mathrm{PV}=$ the maximum mean sprouting recorded at any time during the test) and final mean sprouting (final MDS = cumulative percentage of full sprouting at the end of the test divided by number of days to finish sprouting) were also calculated. SVI index through the present study is calculated by Eq. (2):

$$
\mathrm{SVI}=\mathrm{PV} \times \mathrm{MDS}
$$

\section{Results}

Sprouting and rooting studies of stem cuttings were carried out in three different rooting medium during three seasons (October, June and February) in 2014-2016. The various attributes obtained are given in Figs. 1 and 2.

Fig. 1 gives the VP of stem cuttings in three concentrations used against the non-auxin control. In non-auxin control, no rooting and sprouting were resulted in any of the trials and hence the results were

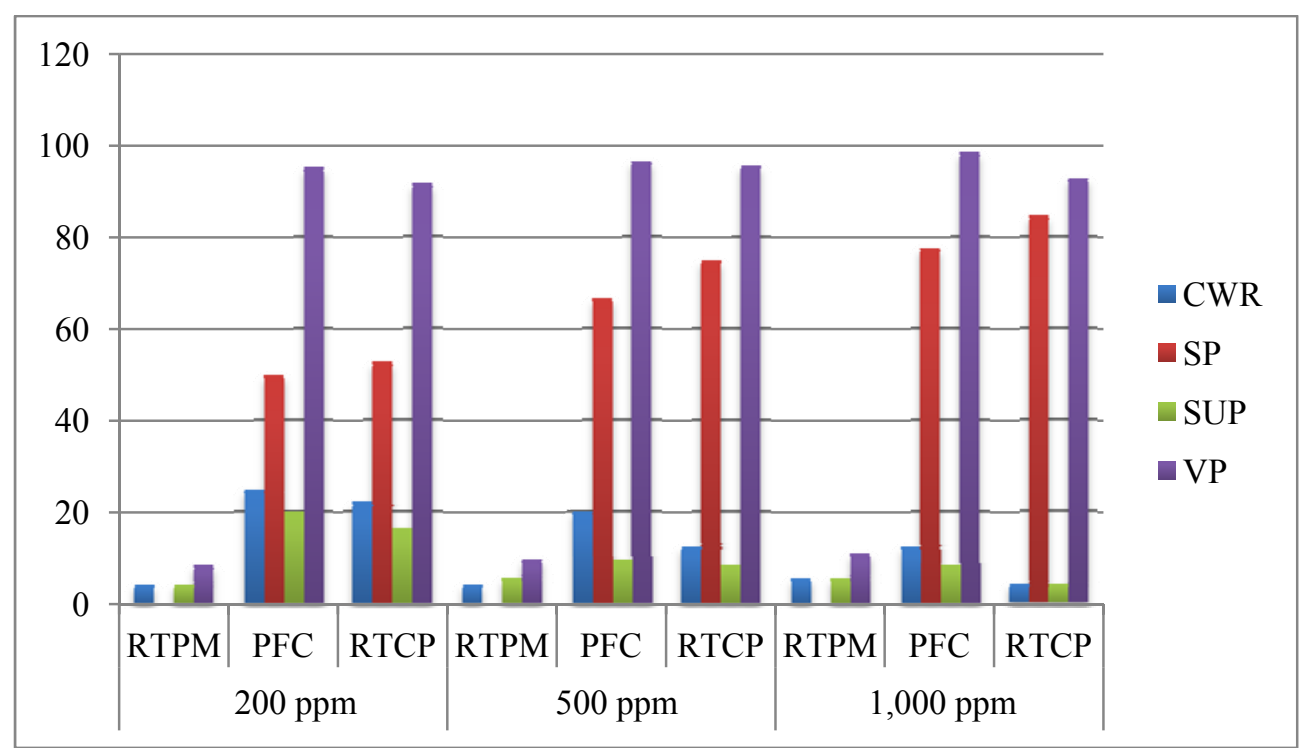

Fig. 1 SP and VP performance (\%) of pink trumpet stem cuttings in different concentrations of IBA and rooting medium. 


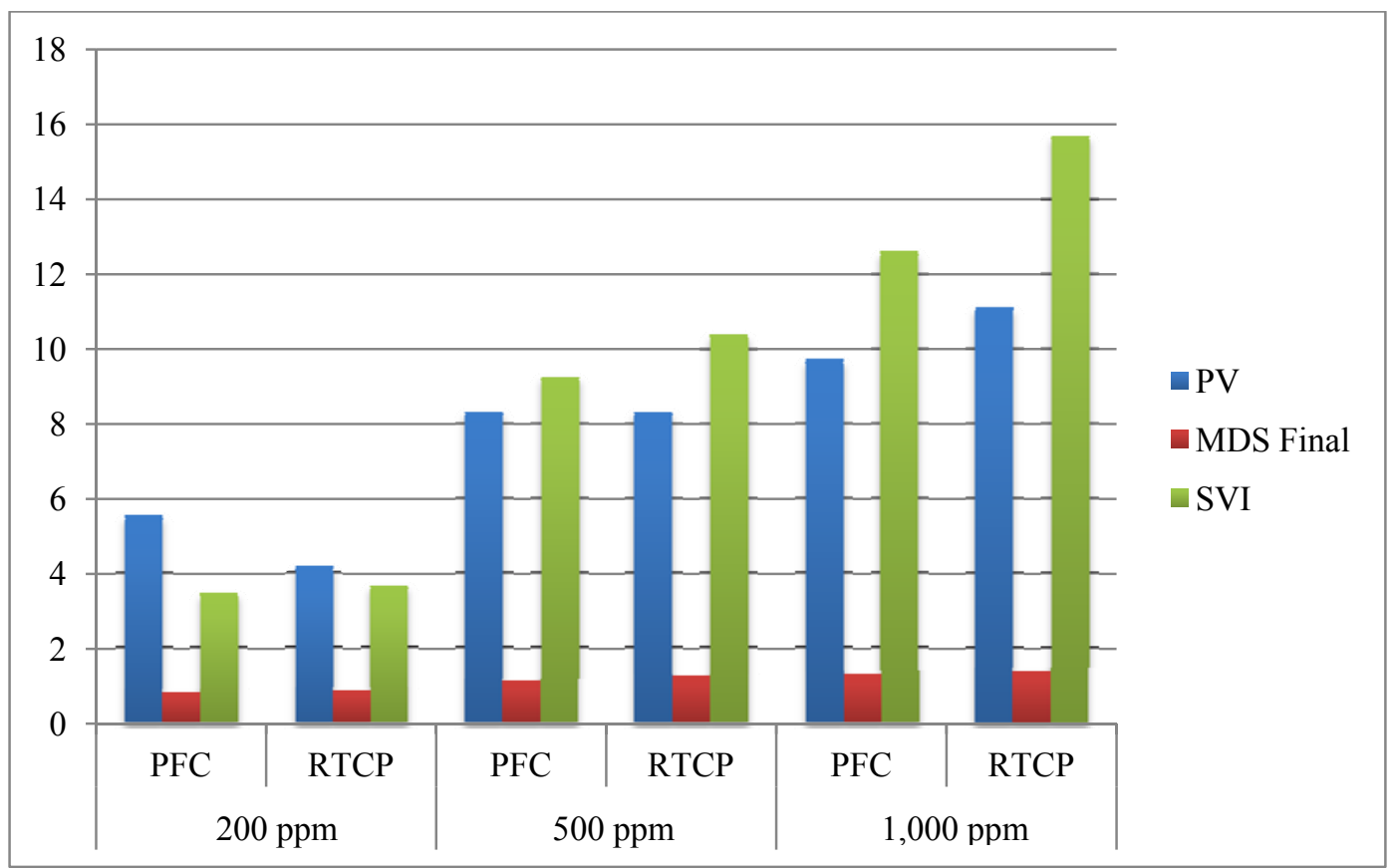

Fig. 2 SVI performance of pink trumpet stem cuttings in different concentrations of IBA and rooting medium.

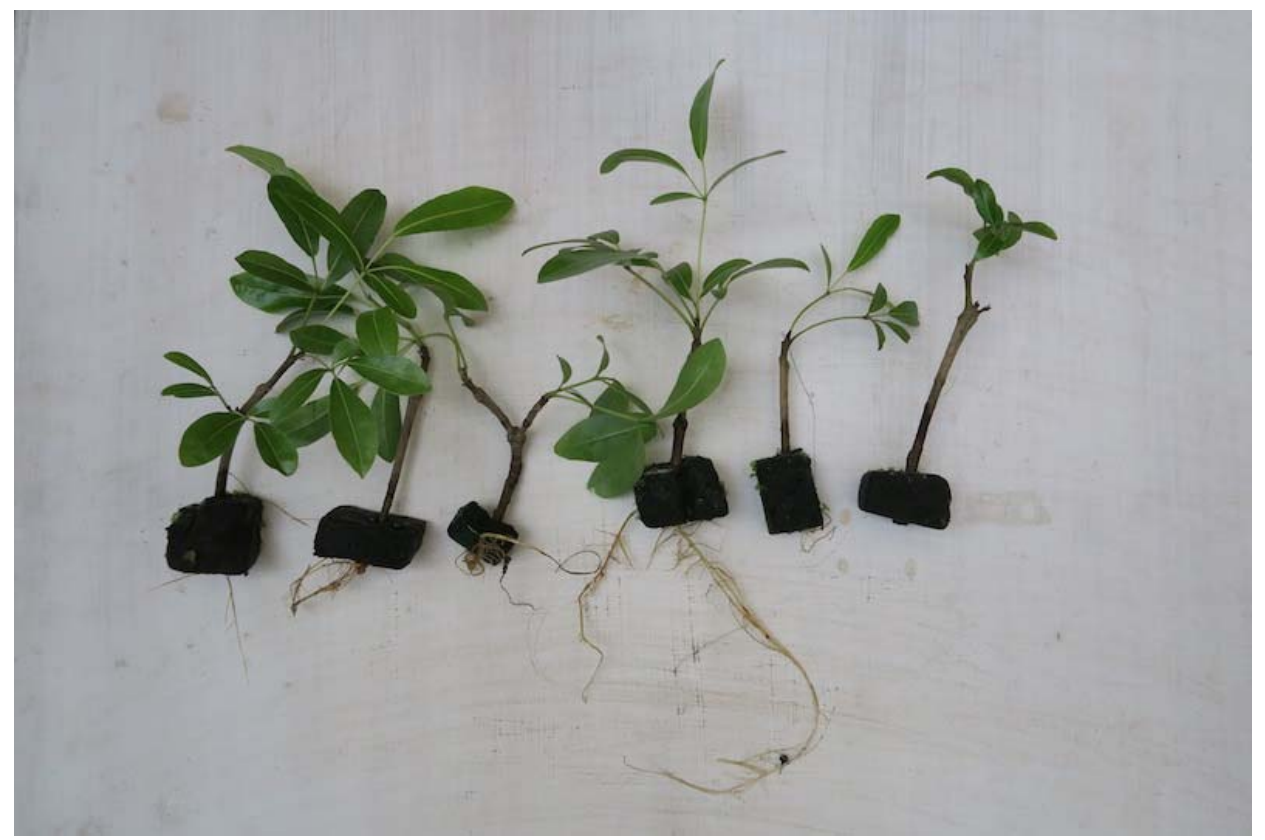

Fig. 3 Rooted stem cuttings of pink trumpet tree in PFC.

taken as zero. Fig. 2 gives SVI in different concentrations. Callus production as well as sprouting and rooting was very poor in the RTPM. VP obtained for IBA treated stem cuttings was high with all the three concentrations used (ranging between $91.66 \%$ and $98.61 \%$ ) in the rooting/sprouting medium PFC (Fig. 3) and RTCP. With stem cuttings of pink trumpet tree, the maximum SVI was obtained in 1,000 ppm treated cuttings. The maximum PV (11.11), MDS final (1.41) and SVI (15.69) was resulted in 1,000 ppm treated cuttings in RTCP. Whereas the lowest PV (4.17), MDS final (0.83) and SVI (3.67) was resulted in $200 \mathrm{ppm}$ IBA treated stem cuttings in PFC. The speed of completion of sprouting/rooting is found 
higher in RTCP than that in PFC.

The ANOVA results on callus formation and callus with root formation show significance at $1 \%$ level between concentrations of IBA.

\section{Discussion}

The SVI study used in the present study is a mathematical approach proposed by Nayagam [16] to determine the quality of rooting material, suitability of the medium used for rooting and the optimum concentration of IBA to be used. Clonal propagation using vegetative method using stem cuttings provides sprouts and shoots throughout the year. Several studies using stem cuttings has been reported [11-15], but treatment of the results with mathematical approach to interpret its efficiency is few.

In field trials, all planting materials gave elite performance in RTCP planting medium. In the trials using 1,000 ppm IBA treated cuttings, SVI was also the highest in RTCP (15.69). Significant differences in rooting were found between various rooting media, such as sand, sawdust and milled pine bark [11-15]. Sprouting percentage and speed of completion of sprouting/rooting initiation could be increased by the use of IBA treatment [16]. Comparing the CWR and SUP percentages, one can also assess the defects in management practices, dormancy and the genotype of the cultivar.

SVI proposed by Nayagam [16] is a modified form of germination value (GV) [6] for seed germination studies. It is also suitable in field and nursery trials for vegetative propagation and IBA treated rooting of cuttings. The incorporation of CWR along with SUP in calculating VP is effective in finding the field oriented defects. SVI and VP are the integrated measure of planting material quality. The speed of sprouting/rooting ability along with the completeness of sprouting can also be determined in vegetative planting materials. Suitable rooting media hold considerably high rooting ability $[17,18]$.

Even though SVI for control were found zero, the
VP for all the IBA treated trials are $>91.66 \%$ (Fig. 1) which indicates that by using alternate methods, like pretreatments and management practices, SVI can be increased. The internal physiology of the planting material may be the reason for the same. The change in planting material and IBA treatment used affect the rooting process, as in the present study, SP was increased in higher concentration (1,000 ppm IBA). Methods for reducing CWR and SUP values can increase SP, and the sample in rooting medium with the least difference between SP and VP will give the maximum performance. The PFC has been found a promising material in rooting experiments, which can reduce the space for rooting trials many folds in the plant nursery. Results of the rooting experiments showed that with very high IBA concentration, rooting ability decreased [17] and hence three concentrations of IBA were used in the present experiment.

\section{Conclusions}

The incorporation of CWR along with SUP in calculating VP makes it suitable for vegetative cultivation practices and it interprets the quality of planting material failure due to management practices and the selection of suitable rooting medium. The PFC has been found a promising material in rooting experiments, which can reduce the space for rooting trials many folds in the plant nursery. This method of planting stock preparation is valuable in large-scale cultivation and much promising in producing quality clonal planting material production in economically important plants in future.

\section{Acknowledgments}

The author expresses his heartfelt gratitude to Dr. Thomas P. Mathew (principal) and Dr. P. M. Kuriachan (head) of the Botany Department, Union Christian College, Aluva, for providing space to conduct field trials in T.C Joseph Memorial Botanical Garden, Department of Botany, Union Christian 
College, Aluva. Also thanks to Mr. Thomachen, gardener of T.C Joseph Memorial Botanical Garden, Department of Botany, Union Christian College, Aluva, for maintaining the field specimens throughout the study period. Extend a word of thanks to Mr. Jabir who helped in statistical correlation.

\section{References}

[1] Harlan, J. R. 1992. Crops and Man, 2nd ed.. Madison: American Society of Agronomy-Crop Science Society.

[2] Solbrig, O. T., and Solbrig, D. J. 1994. So Shall You Reap: Farming and Crops in Human Affairs. Washington, DC: Island Press.

[3] Davies, F. T., Davies, T. M., and Kester, D. E. 1994. "Commercial Importance of Adventitious Rooting to Horticulture.” In Biology of Adventitious Root Formation, edited by Davis, T. M., and Hassing, B. E. US: Springer, 53-60.

[4] Macdonald, A. B. 1986. "Propagation Facilities-Past and Present." Proc. Int. Plant Prop. Soc. 35: 170-5.

[5] Ritchie, G. A. 1994. "Commercial Application of Adventitious Rooting to Forestry." In Biology of Adventitious Root Formation, edited by Davis, T. M., and Hassing, B. E. US: Springer, 37-52.

[6] Czabator, F. J. 1962. "Germination Value: An Index Combining Speed and Completeness of Pine Seed Germination.” Forest Science 8: 386-96.

[7] Nichols, M. A., and Heydecker, W. 1968. "Two Approaches to the Study of Germination Data." Proc. Int. Seed Test. Assoc. 33: 531-40.

[8] Brown, R. F., and Mayer, D. G. 1988. "Representing Cumulative Germination: Part 1, a Critical Analysis of Single-Value Germination Indices.” Annals of Botany 61 (2): 117-25.

[9] Bewley, J. D., and Black, M. 1994. Seeds: Physiology of Development and Germination, 2nd ed.. New York: Plenum Press.
[10] Santana, D. G., Ranal, M. A., Ferreira, W. R., and Mendes-Rodrigues, C. 2004. "Calculating Germination Measurements and Organizing Spreadsheets.” Brazilian Journal of Botany 32 (4): 849-55.

[11] Mesen, F., Newton, A. C., and Leakey, R. R. B. 1997. "Vegetative Propagation of Cordia alliodora (Ruiz \&Pavon) Oken: The Effects of IBA Concentration, Propagation Medium and Cutting Origin." Forest Ecology and Management 92 (1-3): 45-54.

[12] Tchoundjeu, Z., Avana, M. L., Leakey, R. R. B., Simons, A. J., Assah, E., Duguma, B., and Bell. J. M. 2002. "Vegetative Propagation of Prunus africana: Effects of Rooting Medium, Auxin Concentrations and Leaf Area." Agroforestry Systems 54 (3): 183-92.

[13] Ofori, D. A., Newton, A. C., Leakey, R. R. B., and Grace, J. 1996. "Vegetative Propagation of Milicia excelsa by Leafy Stem Cuttings: Effects of Auxin Concentration, Leaf Area and Rooting Medium." Forest Ecology and Management 84 (1-3): 39-48.

[14] Ercisli, S., Esitken, A., Cangi, R., and Sahin, F. 2003. "Adventitious Root Formation of Kiwifruit in Relation to Sampling Date, IBA and Agrobacterium rubi Inoculation." Plant Growth Regulation 41 (2): 133-7.

[15] Akwatulira, F., Gwali, S., Okullo, J. B. L., Ssegawa, P., Tumwebaze, S. B., Mbwambo, J. R., and Muchugi, A. N. 2011. "Influence of Rooting Media and Indole-3-Butyric Acid (IBA) Concentration on Rooting and Shoot Formation of Warburgia ugandensis Stem Cuttings." African J. Plant Sci. 5 (8): 421-9.

[16] Nayagam, J. R. 2015. "Sprouting Value Index: A New Concept in Evaluation of Rooting of Cuttings." World J. Agri. Res. 3 (4): 139-42.

[17] Akakpo, D. B., Amissah, N., Yeboah, J., and Blay, E. 2014. "Effect of Indole 3-Butyric Acid and Media Type on Adventitious Root Formation in Sheanut Tree (Vitellaria paradoxa C. F. Gaertn.) Stem Cuttings." American J. Plant Sci. 5 (3): 313-8.

[18] Nayagam, J. R. 2015. Plantation Technology for Seven Tropical Tree Species. Saarbrücken, Germany: Lambert Academic Publishing (LAP). 